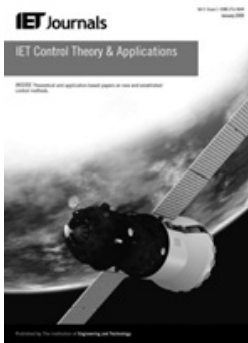

ISSN 1751-8644

\title{
Optimum seeking-based non-linear controller to maximise energy capture in a variable speed wind turbine
}

\author{
$\begin{array}{llll}\text { E. Iyasere } & \text { M. Salah } & \text { D. Dawson } & \text { J. Wagner } \\ & \text { E. Tatlicioglu } & \\ & \end{array}$ \\ ${ }^{1}$ College of Engineering and Science, Clemson University, Clemson, SC, USA \\ ${ }^{2}$ Department of Mechatronics Engineering, Hashemite University, Zarqa, Jordan \\ ${ }^{3}$ Department of Electrical and Electronics Engineering, Izmir Institute of Technology, Urla, Izmir, Turkey \\ E-mail:msalah@hu.edu.jo
}

\begin{abstract}
In this study, an optimum seeking-based robust non-linear controller is proposed to maximise wind energy captured by variable speed wind turbines at low-to-medium wind speeds. The proposed strategy simultaneously controls the blade pitch angle and tip-speed ratio, through the turbine rotor angular speed, to an optimal point at which the power coefficient, and hence the wind turbine efficiency, is maximum. The optimal points are given to the controller by an optimisation algorithm that seeks the unknown optimal blade pitch angle and rotor speed. The control method allows for aerodynamic rotor power maximisation without exact knowledge of the wind turbine model. A representative numerical simulation is presented to show that the wind turbine can be accurately controlled to achieve maximum energy capture.
\end{abstract}

\section{Introduction}

Wind energy has evolved into an attractive energy source for electric utilities, even though it is currently responsible for only $2 \%$ of the global electrical power output. The structure of wind turbines, as well as the fact that the wind energy rate is uncontrollable, compounds the problem of regulating the power capture of the wind turbine. This problem has been alleviated by the construction of variable speed wind turbines; which are designed to regulate the power captured over a range of wind speeds. The efficiency of power regulation is, however, dependent on the selected control method.

Wind turbine control methods include classical techniques $[1,2]$, which utilise a linearised wind turbine system model and a single measured wind turbine output for control. In [1], a PID controller was designed that compensated for wind speed fluctuations by changing the pitch angle to keep the rotor speed constant. The controller was improved by selecting gain values based on minimisation of rotor speed error and the actuator duty cycle. Another common control method is full-state feedback control [3-6], which is sensitive to errors in modelling and measurements. Liebst [4] used individual blade pitch linear quadratic Gaussian optimal control to reduce the loads on a wind turbine owing to environmental factors such as shear and gravity. The dynamics of the wind turbine blade flap, lag and pitch are modelled. Knudsen et al. [3] compared PI and $H_{\infty}$ controllers for regulating the pitch of a $400 \mathrm{~kW}$ wind turbine. The $H_{\infty}$ controller accounts better for turbine model uncertainties as well as error in measuring the wind speed, thus reducing pitch activity. Disturbance accommodating control can account for measurement disturbances by augmenting a state-estimator-based controller to recreate disturbance states using an assumed waveform model. These new states reduce disturbance effects. Wright and Balas [7] described the design of a state space control algorithm for the regulation of the rotor speed of a two-bladed wind turbine in full-load operation using a simple five degreeof-freedom linear model. The authors demonstrated that the pole placement technique can stabilise the turbine model whereas state estimators reduce the number of required measurements. The effects of wind speed fluctuations were reduced by using disturbance accommodating control.

Fuzzy logic control [8-10] and neural networks [11] have been investigated to reduce the uncertainties faced by classical control methods. Prats et al. [9] presented a fuzzy logic application for enhanced energy capture in a variable speed, variable pitch wind turbine. A dynamic model was developed using torque and blade pitch fuzzy control and produced better results than linear control. Zhang et al. [10] compared PID and fuzzy logic control in the control of the rotation of the wind wheel and reverse moment of the generator in a variable speed wind turbine and concluded that fuzzy logic control produce a smoother output with less susceptibility to disturbances. Adaptive control schemes [12-15] have been developed to eliminate some of the problems faced in wind turbine control, such as unknown and time varying model parameters in the wind turbine model. Song et al. [15] used a model reference adaptive control scheme to force a wind turbine with a known power efficiency function, to track a desired rotor 
speed that maximises the energy captured by controlling the excitation winding voltage of the generator. Johnson et al. [13] developed an adaptive control algorithm for controlling the generator torque on a fixed pitch variable speed wind turbine. This approach maximised the energy capture in low-to-medium wind speeds without knowledge of the optimal tip-speed ratio. Moreover, the authors of [16-18] proposed different estimation schemes to estimate the power coefficient in a wind energy conversion system.

In this study, a control strategy is developed to regulate the blade pitch angle and rotor speed of a variable speed wind turbine system. The control objective is to maximise the energy captured by the wind turbine in low-to-medium wind speeds by tracking a desired pitch angle and rotor speed, in the presence of structurally uncertain wind turbine system non-linearities. Additionally, the maximisation of the energy captured is achieved without the knowledge of the relationship that governs the power capture efficiency of the wind turbine. Instead, an optimisation algorithm is developed to seek the unknown optimal blade pitch angle and rotor speed that maximises the energy captured (through the aerodynamic rotor power) while ensuring that the resulting desired trajectories are sufficiently differentiable. The disadvantage of not explicitly knowing the optimal pitch angle and rotor speed a priori is countered by the fact that the optimal rotor speed, and likewise, the optimal pitch angle, will change as the wind speed changes, which can be accounted for by the optimisation algorithm. A robust controller is designed and proven to yield a globally uniformly ultimately bounded (GUUB) stable closed-loop system through Lyapunov-based analysis to maximise energy captured by the wind.

The rest of the paper is organised as follows. In Section 2, a wind turbine dynamic model is presented. In Section 3, a robust non-linear tracking controller is introduced along with the error system dynamics and stability analysis. In Section 4, the development of the system non-linearities estimator is introduced. The reference trajectory generation is discussed in Section 5 utilising an extremum seeking algorithm, followed by numerical simulation results in Section 6. Concluding remarks are presented in Section 7.

\section{Wind turbine dynamic model}

The selected wind turbine model consists of two subsystems [14]: (i) wind turbine blades and pitch actuator, and (ii) drive train that consists of a high-speed shaft, gearbox, low-speed shaft and generator. The aerodynamic power extracted from the wind, $P_{\text {aero }}(t) \in \mathbb{R}^{+}$, can be defined as

$$
P_{\text {aero }} \triangleq \frac{1}{2} C_{p}(\lambda, \beta) \rho A v^{3}
$$

where $\rho \in \mathbb{R}^{+}$is the air density, $A \in \mathbb{R}^{+}$is the rotor swept area, $v(t) \in \mathbb{R}^{+}$is the wind speed, $C_{p}(\cdot) \in \mathbb{R}$ denotes the rotor power coefficient of the wind turbine and it is a function of the tip-speed ratio, $\lambda(t) \in \mathbb{R}^{+}$, and the blade pitch angle, $\beta(t) \in \mathbb{R}$. The tip-speed ratio, $\lambda(t)$, is defined as

$$
\lambda \triangleq \frac{\omega R}{v}
$$

where $\omega(t) \in \mathbb{R}^{+}$is the rotor speed of wind turbine and $R \in$ $\mathbb{R}^{+}$is the rotor radius. In a variable pitch variable speed wind turbine system there exists an optimal rotor speed, denoted by $\omega^{*} \in \mathbb{R}^{+}$(and hence an optimal tip-speed ratio, $\lambda^{*} \in \mathbb{R}^{+}$), and blade pitch angle, denoted by $\beta^{*} \in \mathbb{R}$, for a particular wind speed, $v$, at which the power capture efficiency is maximum. Hence, rotor power coefficient, $C_{p}(\cdot)$, is maximum and represented as $C_{p}^{\max }$ where $C_{p}^{\max }=C_{p}\left(\lambda^{*}, \beta^{*}\right)$ and $\lambda^{*}=\left(\omega^{*} R / v\right)$. The aerodynamic power captured by the rotor, $P_{\text {aero }}(t)$, can also be expressed as

$$
P_{\text {aero }}=\tau_{\text {aero }} \omega
$$

where $\tau_{\text {aero }}(t) \in \mathbb{R}^{+}$is the aerodynamic torque applied to the rotor by the wind. An expression for $\tau_{\text {aero }}(t)$ can be derived from (1) to (3) as

$$
\tau_{\text {aero }}=\frac{1}{2} \rho A R \frac{C_{p}(\lambda, \beta)}{\lambda} v^{2}
$$

Remark 1: Since the rotor power coefficient, $C_{p}(\cdot)$, is eventually unknown, hence the aerodynamic torque, $\tau_{\text {aero }}(t)$, is unmeasurable.

The wind turbine model structure can be written as $[14,19]$

$$
M \ddot{X}+f(\beta, \dot{X}, v)=\tau_{\mathrm{c}}
$$

where

$$
X(t) \triangleq\left[\int_{t_{0}}^{t} \omega(\sigma) \mathrm{d} \sigma, \quad \beta(t)\right]^{\mathrm{T}} \in \mathbb{R}^{2}
$$

are the state variables, $M \in \mathbb{R}^{2 \times 2}$ denotes the lumped inertia matrix, $f(\cdot) \triangleq\left[-\tau_{\text {aero }}(t), \quad N(\cdot)\right]^{\mathrm{T}} \in \mathbb{R}^{2}$ represents the wind turbine system uncertain non-linearities, $N(\cdot) \in \mathbb{R}$ designates the pitch subsystem uncertain non-linearities and $\tau_{\mathrm{c}}(t) \in \mathbb{R}^{2}$ is the control input torques for the generator and the pitch actuator.

To facilitate the control development process, six assumptions are imposed.

Assumption 1: The system parameters $R, A$ and $\rho$ are assumed to be known constants.

Assumption 2: The lumped inertia matrix, $M$, is assumed to be a known symmetric and positive-definite matrix.

Assumption 3: The variables $v(t), \omega(t), \beta(t)$ and $\dot{\beta}(t)$ are assumed to be measurable.

Assumption 4: The wind velocity, $v(t)$, is assumed to be constant or slowly time varying.

Assumption 5: The functions $f(\cdot), \dot{f}(\cdot)$ and $\ddot{f}(\cdot)$ are assumed to be bounded provided that their arguments are bounded.

Remark 2: The Euclidean norm of the function vector $f(\beta, \dot{X}, v)$ can be upper bounded by a known function such that $\|f(\beta, \dot{X}, v)\| \leq \rho_{z}(\beta, \dot{X})$.

\section{Non-linear controller design}

The control objective is to maximise the aerodynamic power extracted from the wind, $P_{\text {aero }}(t)$, while tracking a desired rotor speed, $\omega_{\mathrm{d}}(t) \in \mathbb{R}^{+}$, and blade pitch angle, $\beta_{\mathrm{d}}(t) \in \mathbb{R}$, such that $\omega(t) \rightarrow \omega_{\mathrm{d}}(t)$ and $\beta(t) \rightarrow \beta_{\mathrm{d}}(t)$ as $t \rightarrow \infty$. To quantify this control objective, measurement tracking errors, denoted by $e_{1}(t), e_{2}(t) \in \mathbb{R}$, are defined as

$$
\begin{aligned}
& e_{1} \triangleq \omega_{\mathrm{d}}-\omega \\
& e_{2} \triangleq \beta_{\mathrm{d}}-\beta
\end{aligned}
$$


Remark 3: The variables $\omega_{\mathrm{d}}(t)$ and $\beta_{\mathrm{d}}(t)$ are designed and updated online using a numerical-based two-dimensional optimisation algorithm to maximise the rotor power, $P_{\text {aero }}(t)$, such that $\beta_{\mathrm{d}}(t) \rightarrow \beta^{*}$ and $\omega_{\mathrm{d}}(t) \rightarrow \omega^{*}$ at a given wind velocity, $v$, hence $P_{\text {aero }}(t) \rightarrow P_{\max }$ where $P_{\max } \triangleq$ $(1 / 2) C_{p}^{\max } \rho A v^{3}$, and $\left[\omega^{*}, \beta^{*}\right]^{\mathrm{T}}$ denotes the set of optimal values resulting from the extremum seeking algorithm. In addition, $X_{\mathrm{d}}(t) \triangleq\left[\int_{t_{0}}^{t} \omega_{\mathrm{d}}(\sigma) \mathrm{d} \sigma, \quad \beta_{\mathrm{d}}(t)\right]^{\mathrm{T}}$ is designed such that $\beta_{\mathrm{d}}(t), \dot{\beta}_{\mathrm{d}}(t), \ddot{\beta}_{\mathrm{d}}(t), \dddot{\beta}_{\mathrm{d}}(t), \omega_{\mathrm{d}}(t), \dot{\omega}_{\mathrm{d}}(t)$ and $\ddot{\omega}_{\mathrm{d}}(t)$ are bounded.

The following filtered tracking error, denoted by $r_{2}(t) \in$ $\mathbb{R}$, is defined to facilitate the subsequent controller design

$$
r_{2} \triangleq \dot{e}_{2}+\mu e_{2}
$$

where $\mu \in \mathbb{R}^{+}$is a control gain.

Remark 4: Based on the definition of $r_{2}(t)$ in (7), standard arguments can be utilised to prove that if $r_{2}(t)$ is bounded, then $e_{2}(t)$ and $\dot{e}_{2}(t)$ are bounded.

After defining a composite error signal denoted by $z(t) \triangleq\left[e_{1}(t), \quad r_{2}(t)\right]^{\mathrm{T}} \in \mathbb{R}^{2}$, the following expression can be obtained by taking its first time derivative and premultiplying the resulting expression by $M$

$$
\begin{aligned}
& M \dot{z}=M\left[\begin{array}{l}
\dot{e}_{1} \\
\ddot{e}_{2}
\end{array}\right]+M\left[\begin{array}{c}
0 \\
\mu \dot{e}_{2}
\end{array}\right] \\
& M \dot{z}=M \ddot{X}_{\mathrm{d}}-M \ddot{X}+M\left[\begin{array}{c}
0 \\
\mu \dot{e}_{2}
\end{array}\right] \\
& M \dot{z}=M \ddot{X}_{\mathrm{d}}+f-\tau_{\mathrm{c}}+M\left[\begin{array}{c}
0 \\
\mu \dot{e}_{2}
\end{array}\right]
\end{aligned}
$$

where (5)-(7) were utilised. Based on the subsequent stability analysis and the structure of the open-loop error system in (10), the control input $\tau_{\mathrm{c}}(t)$ is designed as

$$
\tau_{\mathrm{c}}=M \ddot{X}_{\mathrm{d}}+M\left[\begin{array}{c}
0 \\
\mu \dot{e}_{2}
\end{array}\right]+\hat{f}_{s}+K z+\frac{\rho_{z}^{2}}{\varepsilon} z
$$

where $\hat{f}_{s}(\cdot)$ is defined as $\hat{f}_{s}(\cdot) \triangleq\left(1 / \tau_{1} s+1\right) \operatorname{sat}\{\hat{f}(\cdot)\}, \hat{f}(\cdot)$ is the estimate of $f(\cdot)$ and will be designed in Section 4 , and $\tau_{1} \in \mathbb{R}^{+}$is a small constant, $s$ is the Laplace variable, sat $\{\cdot\}$ is the standard saturation function, $K \in \mathbb{R}^{+}$is a control gain, $\varepsilon \in \mathbb{R}^{+}$is a small constant and $\rho_{z}(\beta, \dot{X})$ was introduced in Remark 2 .

Remark 5: The variables $\hat{f}_{s}(\cdot)$ and $\dot{\hat{f}}_{s}(\cdot)$ are bounded since the output of a saturation function is always bounded and $\left(1 / \tau_{1} s+1\right)$ is a proper bounded filter. Thus, it may be assumed that $\left\|\hat{f}_{s}(\cdot)\right\| \leq \rho_{N}$, where $\rho_{N} \in \mathbb{R}^{+}$is a bounding constant.

Substituting the control torque from (11) into the openloop dynamics of (10) results in the following closed-loop error system

$$
M \dot{z}=f-\hat{f}_{s}-K z-\frac{\rho_{z}^{2}}{\varepsilon} z
$$

where the function $\rho_{z}(\beta, \dot{X})$ was introduced in Remark 2 . A Lyapunov stability analysis guarantees that the system described in (5) will be stable when applying the control law introduced in (12).

Theorem 1: The controller given in (12) ensures that: (i) all closed-loop signals stay bounded for all time; and (ii) tracking is GUUB.

\section{Proof: See Appendix 1.}

\section{Estimation of system non-linearities}

As previously stated, the main control objective is to maximise the aerodynamic power captured by the rotor of a variable speed wind turbine with structurally uncertain system non-linearities. The control development in Section 3 requires that the system non-linearities be estimated. The estimate of $f(\cdot)$, denoted by $\hat{f}(\cdot)$, is developed for two reasons: (i) $\hat{f}(\cdot)$ is used as a feedforward term in the control design, through $\hat{f}_{s}(\cdot)$ to reduce the magnitude of the control input torque, $\tau_{\mathrm{c}}(t)$, (ii) from Remark 1 and (3), $P_{\text {aero }}(t)$ is unavailable for measurement and by utilising $\hat{f}(\cdot)=$ $\left[\begin{array}{ll}-\hat{\tau}_{\text {aero }}(\cdot), & \hat{N}(\cdot)\end{array}\right]^{\mathrm{T}}$, an estimate of the captured aerodynamic power, $\hat{P}_{\text {aero }}(t)$, can be realised where $\hat{P}_{\text {aero }}(t)=\hat{\tau}_{\text {aero }}(t) \omega(t)$.

Now let us consider the two systems

$$
\begin{aligned}
M \ddot{X} & =\tau_{\mathrm{c}}-f \\
M \ddot{\hat{X}} & =\tau_{\mathrm{c}}-\hat{f}
\end{aligned}
$$

where $\hat{X}(t) \in \mathbb{R}^{2}$ denotes the estimate of the states, and $\hat{f}(\cdot)$ is the estimate of $f(\cdot)$. The objective of the estimator is to track the system uncertain non-linearities, $f(\cdot)$, such that $\hat{f}(\cdot) \rightarrow f(\cdot)$ as $t \rightarrow \infty$. To quantify this objective, the observation errors, $\dot{\tilde{X}}(t), \tilde{f}(t) \in \mathbb{R}^{2}$ are defined as

$$
\begin{gathered}
\dot{\tilde{X}} \triangleq \dot{\hat{X}}-\dot{X} \\
\tilde{f} \triangleq \hat{f}-f
\end{gathered}
$$

The filtered observation error, denoted by $r(t) \in \mathbb{R}^{2}$, is defined to facilitate the subsequent estimator design

$$
r \triangleq \ddot{\tilde{X}}+\Delta \dot{\tilde{X}}
$$

where $\Delta \in \mathbb{R}^{+}$is an observer gain. After taking the first time derivative of (17) and pre-multiplying by $M$ it may be shown that

$$
M \dot{r}=M \ddot{\tilde{X}}+\Delta M \ddot{\tilde{X}}=-\dot{\tilde{f}}+\Phi-\dot{\tilde{X}}
$$

where $\Phi(\cdot) \triangleq \Delta M \ddot{\tilde{X}}+\dot{\tilde{X}}$ and (13)-(16) were utilised.

Remark 6: The auxiliary signal $\Phi(\bullet)$ can be upper bounded such that $\|\Phi(\cdot)\| \leq \bar{\rho}_{N}\|\bar{z}\|$ where $\bar{z}(t) \triangleq[\tilde{\omega}(t), \quad r(t)]^{\mathrm{T}} \in \mathbb{R}^{3}$ and $\bar{\rho}_{N} \in \mathbb{R}^{+}$is a bounding constant. $\tilde{\omega}(t) \triangleq \hat{\omega}(t)-\omega(t)$ and $\hat{\omega}(t) \in \mathbb{R}^{+}$is the estimate of the rotor speed.

Based on the structure of (18) as well as the subsequent stability analysis, the following implementable continuous estimator is proposed to achieve the stated estimator objective

$$
\dot{\hat{f}}=(k+\Delta) r+\rho_{0} \operatorname{sgn}(\dot{\tilde{X}})
$$

where $k, \rho_{0} \in \mathbb{R}^{+}$are control gains. Before presenting the stability analysis, the following lemma will be introduced and later invoked. 
Lemma 1: Let the auxiliary function $L(t) \in \mathbb{R}$ be defined as

$$
L \triangleq r^{\mathrm{T}}\left(\dot{f}-\rho_{0} \operatorname{sgn}(\dot{\tilde{X}})\right)
$$

If the control gain $\rho_{0}$ is selected to satisfy the sufficient condition

$$
\rho_{0}>\|\dot{f}(\cdot)\|+\frac{\|\ddot{f}(\cdot)\|}{\Delta}, \quad \text { then } \int_{t_{0}}^{t} L(\tau) \mathrm{d} \tau \leq \zeta_{0}
$$

where the constant $\zeta_{0} \in \mathbb{R}^{+}$is defined as

$$
\zeta_{0} \triangleq \rho_{0}\left\|\dot{\tilde{X}}\left(t_{0}\right)\right\|_{1}+\dot{\tilde{X}}^{\mathrm{T}}\left(t_{0}\right) \dot{f}\left(t_{0}\right)
$$

Proof: See Appendix 2.

Theorem 2: The estimator in (19) ensures that asymptotic tracking is obtained, in the sense that $\|\dot{\tilde{X}}(t)\|,\|\tilde{\tilde{X}}(t)\|$, $\|r(t)\| \rightarrow 0$ as $t \rightarrow \infty$.

Proof: See Appendix 3.

\section{Trajectory generation and extremum seeking algorithm}

In Remark 3, it was assumed that a composite set of desired trajectories defined as $\xi_{\mathrm{d}}(t) \triangleq\left[\omega_{\mathrm{d}}(t), \quad \beta_{\mathrm{d}}(t)\right]^{\mathrm{T}}$ can be generated such that $\xi_{\mathrm{d}}(t), \dot{\xi}_{\mathrm{d}}(t), \ddot{\xi}_{\mathrm{d}}(t)$ and $\dddot{\beta}_{\mathrm{d}}(t)$ are bounded and $\xi_{\mathrm{d}}(t) \rightarrow \xi^{*}$ where $\xi^{*}$ is an unknown set of constants that maximise the aerodynamic rotor power, $P_{\text {aero }}(t)$, at a given wind speed, $v(t)$. As stated previously, $P_{\text {aero }}(t)$ is unmeasurable, thus the estimated captured power $\hat{P}_{\text {aero }}=\hat{\tau}_{\text {aero }} \omega$ can be used instead. The extremum seeking algorithm used in this study is the Powell's method [20]. Powell's method only requires measurement of the output function, $\hat{P}_{\text {aero }}(t)$, and an initial guess (not required to be close to the value of $\xi^{*}$ ). Powell's method can then find $\xi^{*}$ by performing a series of one-dimensional line maximisations (using Brent's method) with convergence because of the non-trivial choice of search directions [21] (new directions are calculated using the extended parallel subspace property to avoid linear dependence).

To ensure that $\xi_{\mathrm{d}}(t), \dot{\xi}_{\mathrm{d}}(t), \ddot{\xi}_{\mathrm{d}}(t)$ and $\dddot{\beta}_{\mathrm{d}}(t)$ are bounded, a filter-based form of Powell's method is used, wherein at each iteration, $\xi_{\mathrm{d}}(i)$ is passed through a set of third-order stable and proper low-pass filters to generate continuous bounded signals for $\xi_{\mathrm{d}}(t), \dot{\xi}_{\mathrm{d}}(t), \ddot{\xi}_{\mathrm{d}}(t)$ and $\dddot{\beta}_{\mathrm{d}}(t)$ where $i=$ $\mathbb{Z}^{+}$. The filters shown in (22)-(25) are used in this study, where $\zeta_{1}, \zeta_{2}, \zeta_{3}, \zeta_{4} \in \mathbb{R}^{+}$are filter constants. The optimisation algorithm waits until certain error thresholds are met before making the next guess (i.e. if $\left\|\xi_{\mathrm{d}}(t)-\xi_{\mathrm{d}}(i)\right\| \leq \bar{e}_{1},|\tilde{f}(\cdot)| \leq$ $\bar{e}_{2}$ and $\left\|\xi(t)-\xi_{\mathrm{d}}(t)\right\| \leq \bar{e}_{3}$, then $i=i+1$ where $\bar{e}_{1}, \bar{e}_{2}, \bar{e}_{3} \in$ $\mathbb{R}^{+}$are some pre-defined threshold constants and $i=\mathbb{Z}^{+}$):

$$
\begin{aligned}
\xi_{\mathrm{d}}(t) & =\frac{\zeta_{1}}{s^{3}+\zeta_{2} s^{2}+\zeta_{3} s+\zeta_{4}} \xi_{\mathrm{d}}(i) \\
\dot{\xi}_{\mathrm{d}}(t) & =\frac{\zeta_{1} s}{s^{3}+\zeta_{2} s^{2}+\zeta_{3} s+\zeta_{4}} \xi_{\mathrm{d}}(i) \\
\ddot{\xi}_{\mathrm{d}}(t) & =\frac{\zeta_{1} s^{2}}{s^{3}+\zeta_{2} s^{2}+\zeta_{3} s+\zeta_{4}} \xi_{\mathrm{d}}(i) \\
\dddot{\beta}_{\mathrm{d}}(t) & =\frac{\zeta_{1} s^{3}}{s^{3}+\zeta_{2} s^{2}+\zeta_{3} s+\zeta_{4}} \beta_{\mathrm{d}}(i)
\end{aligned}
$$

\section{Simulation results}

A numerical simulation is presented to illustrate the performance of the controller introduced in (11), and to demonstrate the numerical-based extremum seeking reference trajectory generator. The simulation was performed for a simple case where the wind speed was set to be a constant (e.g. $v=1.5 \mathrm{~m} / \mathrm{s}^{2}$ ) and the system model in (5), corresponded to a small turbine, was assumed to have the following system non-linearities

$$
f(\cdot)=\left[-\frac{1}{2} \rho A \frac{C_{p}(\lambda, \beta)}{\omega} v^{3}, \quad 0\right]^{\mathrm{T}}
$$

and the model parameters are listed in Table 1.

The control gains were tuned to provide the best response for the wind turbine system and selected to be $K=10$, $k=10, \mu=3, \Delta=2, \tau_{1}=1$ and $\varepsilon=0.1$. The filter constants were chosen to be $\zeta_{1}=81, \zeta_{2}=12, \zeta_{3}=54$ and $\zeta_{4}=108$. The desired and actual rotor speeds, $\omega_{\mathrm{d}}(t)$ and $\omega(t)$, respectively, are shown in Fig. 1. It is clear that $\omega(t)$ successfully tracks $\omega_{\mathrm{d}}(t)$. Similarly, it is clear that $\beta(t)$ successfully tracks $\beta_{\mathrm{d}}(t)$ as shown in Fig. 2 . The power coefficient function $C_{p}(\lambda, \beta)$, illustrated in Fig. 3, was obtained using blade-element momentum theory in [22]. For this case, $C_{p}^{\max }=0.4405$ at $\left[\lambda^{*}=8, \beta^{*}=2.4\right]$ which, according to $(2)$, corresponds to $\left[\omega^{*}=6, \beta^{*}=2.4\right]$. The numerical-based extremum seeking algorithm converged to $\left[\omega^{*}=6.075, \beta^{*}=2.3\right]$ as shown in Figs. 1 and 2 and the maximum simulated power coefficient $C_{p}(t)$ converges to $C_{p}^{\max }=0.4401$ as shown in Fig. 4 and that was satisfactory.

After analysis, the following four conclusions can be made. First, from Figs. 2 and 3, it can be concluded that $\omega(t) \rightarrow \omega_{\mathrm{d}}(t), \beta(t) \rightarrow \beta_{\mathrm{d}}(t)$ and $\omega_{\mathrm{d}}(t) \rightarrow \omega^{*}, \beta_{\mathrm{d}}(t) \rightarrow \beta^{*} ;$

Table 1 Values of the system parameters used in the numerical simulation

\begin{tabular}{lcccc}
\hline Variables & $M$ & $R$ & $A$ & $\rho$ \\
\hline Value & {$\left[\begin{array}{ll}5 & 0 \\
0 & 5\end{array}\right]$} & 2 & 12.6 & 1.2 \\
Units & $\mathrm{kg} \mathrm{m}^{2}$ & $\mathrm{~m}$ & $\mathrm{~m}^{2}$ & $\mathrm{~kg} / \mathrm{m}^{3}$ \\
\hline
\end{tabular}

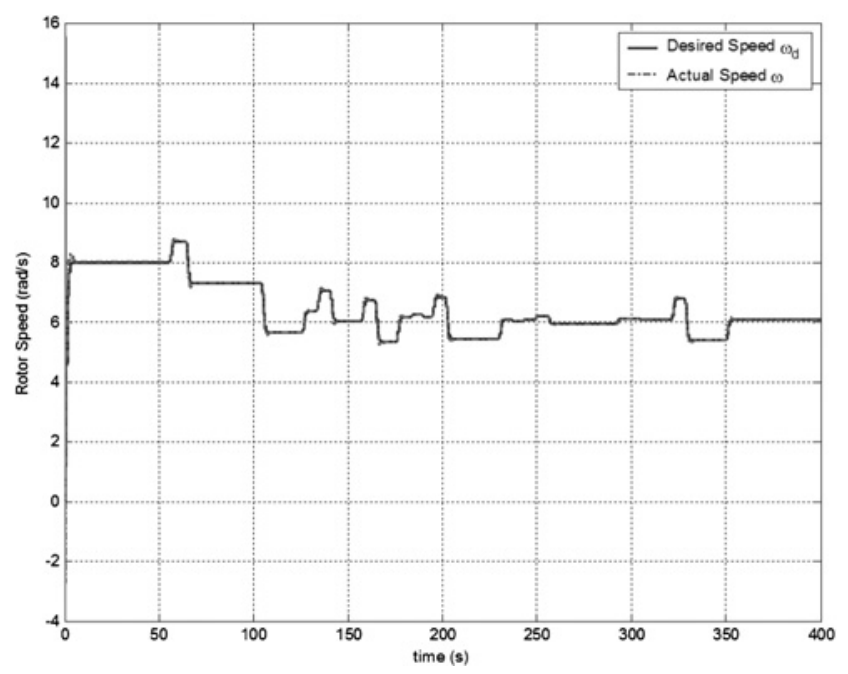

Fig. 1 Desired rotor speed, $\omega_{\mathrm{d}}(t)$, against actual rotor speed, $\omega(t)$ 


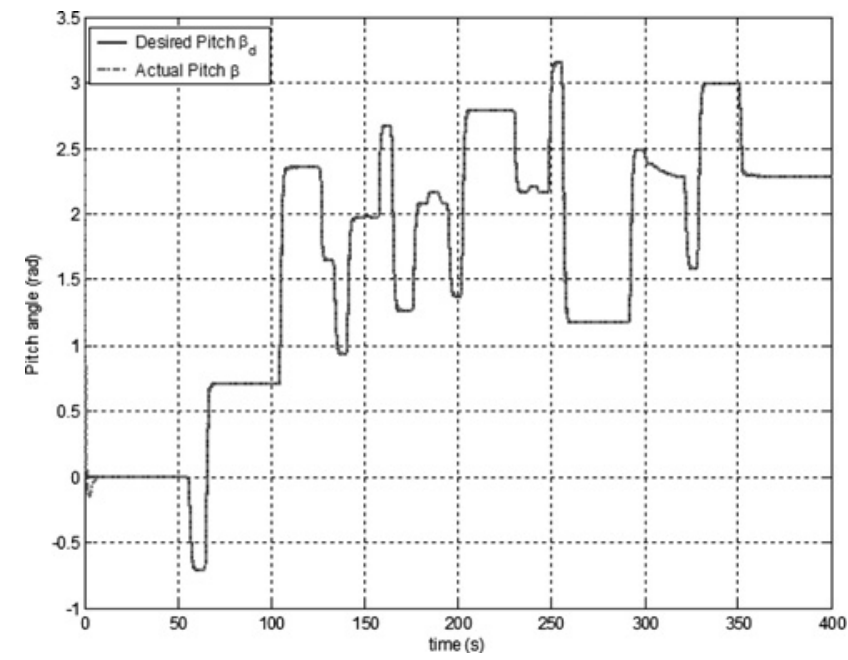

Fig. 2 Desired blade pitch angle, $\beta_{\mathrm{d}}(t)$, against actual blade pitch angle, $\beta(t)$

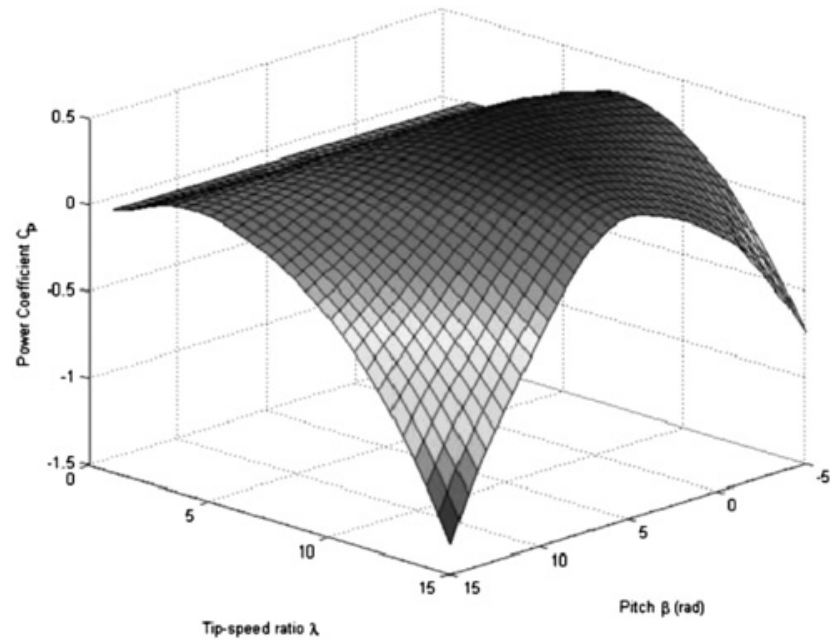

Fig. 3 Power coefficient function, $C_{p}(t)$, against tip-speed ratio, $\lambda(t)$, and blade pitch angle, $\beta(t)$, for the simulated wind turbine

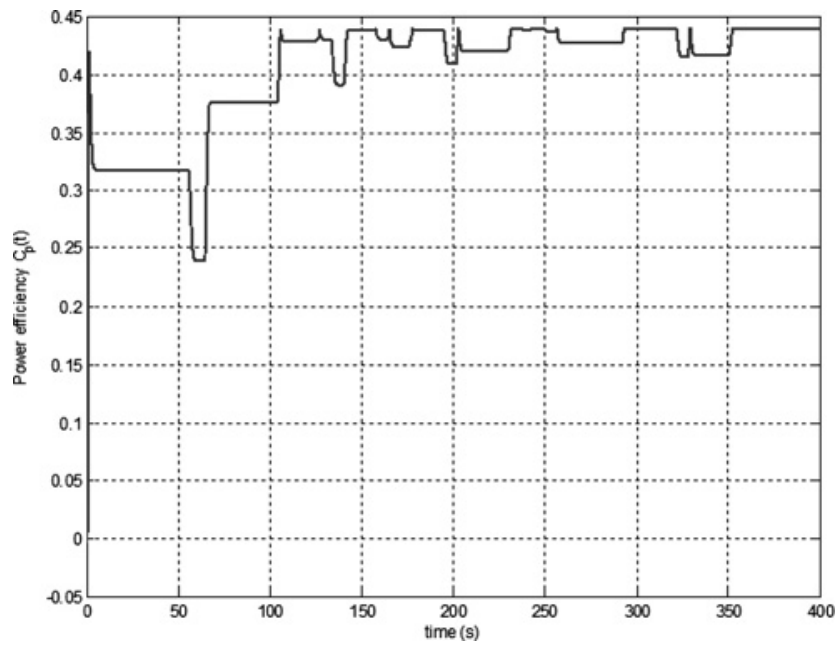

Fig. 4 Maximum rotor power coefficient, $C_{p}(t)$, resulting from the numerical optimisation algorithm
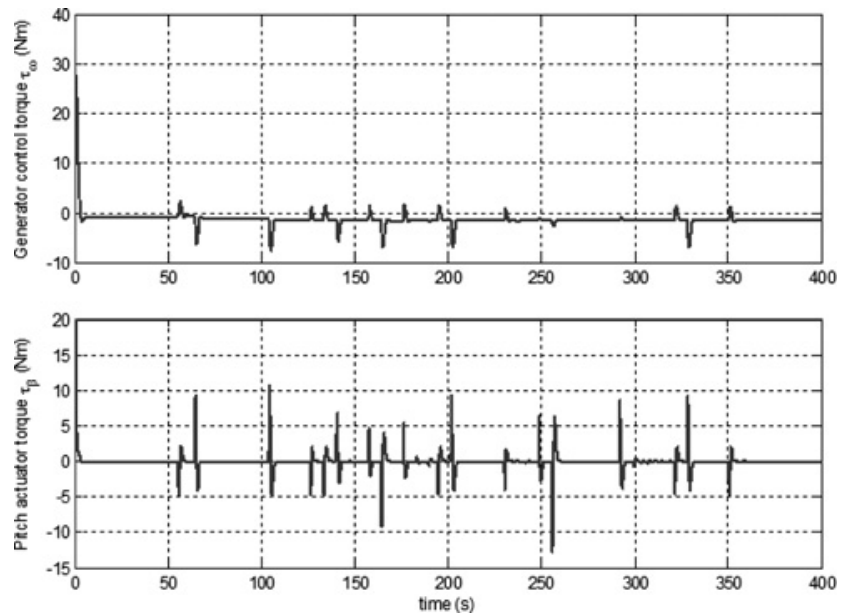

Fig. 5 Simulated (top plot) control torque for drive train subsystem, $\tau_{\omega}(t)$, and (bottom plot) control torque for pitch subsystem, $\tau_{\beta}(t)$

thus $\omega(t) \rightarrow \omega^{*}, \beta(t) \rightarrow \beta^{*}$ which fulfils the stated control objective. Second, the results of the extremum seeking algorithm were within $5 \%$ of the nominal optimum blade pitch angle and rotor speed. Next, the tracking errors, $e_{1}(t)$ and $e_{2}(t)$, for both subsystems settle to a neighbourhood of $\pm 5 \times 10^{-6}$ around zero after $400 \mathrm{~s}$. Finally, the control input, $\tau_{\mathrm{c}}(t)$, is bounded as shown in Fig. 5. Overall, the control strategy proposed in this study produced favourable results and demonstrates that at low-to-medium speeds, it is possible to attain optimal power capture efficiency in variable speed variable pitch wind turbines in the presence of structural uncertainty in the form of unknown system non-linearities. Additionally, the robustness of the control strategy allows for varying wind speeds in obtaining the global maximal power efficiency.

\section{Conclusions}

A non-linear controller has been developed for a variable speed wind turbine system to optimise the energy captured by the wind turbine. A desired blade pitch angle and rotor speed trajectory generator is provided that seeks the unknown optimal set-point while ensuring the trajectory remains bounded and sufficiently differentiable. To track the desired trajectory, a robust controller is developed, which is proven to yield a GUUB stable closed-loop system through Lyapunov-based analysis. The simulation results demonstrated the excellent performance of the robust controller and the numerical-based extremum seeking algorithm.

\section{References}

1 Malinga B., Sneckenberger J., Feliachi J.: 'Modeling and control of a wind turbine as a distributed resource'. Proc. 35th Southeastern Symp. on System Theory, Morgantown, WV, 2003, pp. 108-112

2 Svensson J., Ulen E.: 'The control system of WTS-3 instrumentation and testing'. Proc. Fourth Int. Symp. on Wind Energy Systems, Stockholm, Sweden, 1982, pp. 195-215

3 Knudsen T., Andersen P., Tiffner-Clausen S.: 'Comparing PI and robust control pitch controllers on a $400 \mathrm{KW}$ wind turbine by full scale tests'. Technical report R-97-4174, Department of Control Engineering, Aalborg University, Aalborg, Denmark, 1997

4 Liebst B.: 'Pitch control system for large-scale wind turbines', J. Energy, 1983, 7, (2), pp. 182-192 
5 Mattson S.: 'Modeling and control of large horizontal axis wind power plants'. PhD thesis, Lund Institute of Technology, Lund, Sweden, 1984

6 Stol K., Balas M.: 'Full-state feedback control of a variable-speed wind turbine: a comparison of periodic and constant gains', IEEE J. Sol. Energy Eng., 2011, 123, (4), pp. 319-326

7 Wright A., Balas M.: 'Design of state-space-based control algorithms for wind turbine speed regulation', IEEE J. Sol. Energy Eng., 2003, 125, (4), pp. 386-395

8 Chedid R., Karaki S., El-Chamali C.: 'Adaptive fuzzy control for wind-diesel weak power systems', IEEE Trans. Energy Convers., 2000, 15, (1), pp. 71-78

9 Prats M., Carrasco J., Galvan E., Sanchez J., Franquelo L., Batista C.: 'Improving transition between power optimization and power limitation of variable speed, variable pitch wind turbines using fuzzy control techniques'. Proc. IEEE Int. Conf. on Industrial Electronics, Control, and Instrumentation (IECON), Nagoya, Japan, 2000, vol. 3, pp. 1497-1502

10 Zhang X., Wang W., Liu Y.: 'Fuzzy control of variable speed wind turbine'. Proc. Sixth World Congress on Intelligent Control and Automation, Dalian, China, 2006, pp. 3872-3876

11 Kanellos F., Hatziargyriou N.: 'A new control scheme for variable speed wind turbines using neural networks'. Proc. IEEE Power Engineering Society Transmission and Distribution Conf., New York, 2002, pp. 360-365

12 Freeman J., Balas M.: 'An investigation of variable speed horizontalaxis wind turbines using direct model-reference adaptive control'. Proc. 18th ASME Wind Energy Symp., Reno, NV, 1999, pp. 66-76

13 Johnson K., Fingersh L., Balas M., Pao L.: 'Methods for increasing region 2 power capture on a variable-speed wind turbine', ASME J. Sol. Energy Eng., 2004, 126, (4), pp. 1092-1100

14 Johnson K., Pao L., Balas M., Fingersh L.: 'Control of variable-speed wind turbines: standard and adaptive techniques for maximizing energy capture', IEEE Control Syst. Mag., 2006, 26, (3), pp. 70-81

15 Song Y., Dhinakaran B., Bao X.: 'Variable speed control of wind turbines using nonlinear and adaptive algorithms', J. Wind Eng. Ind. Aerodyn., 2000, 85, (3), pp. 293-308

16 Busawon K., Jovanovic M., Dodson L.: 'Power coefficient estimation in a wind energy conversion system'. Proc. 12th Int. Power Electronics and Motion Control Conf., Portoroz, Slovenia, 2006, pp. 1873-1877

17 Dodson L., Busawon K., Jovanovic M.: 'Estimation of the power coefficient in a wind conversion system'. Proc. 44th IEEE Conf. on Decision and Control, and the European Control Conf., Seville, Spain, 2005, pp. 3450-3455

18 Odgaard P., Damgaard C., Nielsen R.: 'On-line estimation of wind turbine power coefficients using unknown input observers'. Proc. 17th IFAC World Congress, Seoul, Korea, 2008, pp. 10646-10651

19 Dai1 J.-C., Hu Y.-P., Liu D.-S., Long X.: 'Modelling and characteristics analysis of the pitch system of large scale wind turbines', J. Mech. Eng. Sci., 2011, 225, (3), pp. 558-567

20 Vanderplaats G.: 'Numerical optimization techniques for engineering design: with applications' (McGraw-Hill College, NY, 1984, 1st edn.)

21 Reklaitis G., Ravindran A., Ragsdell K.: 'Engineering optimization' (John Wiley \& Sons, Hoboken, NJ, 1983), pp. 41-130

22 NWTC design codes (WT_Perf by Marshall Buhl), http://wind. nrel.gov/designcodes/simulators/wtperf/, 1 August 2007

23 Kokotovic P.: 'The joy of feedback: nonlinear and adaptive', IEEE Control Syst. Mag., 1992, 12, (3), pp. 7-17

24 Slotine J., Li W.: 'Applied nonlinear control' (Prentice Hall, Englewood Cliffs, NJ, 1991)

\section{Appendix 1: Proof of Theorem 1}

A non-negative function, denoted by $V(z(t)) \in \mathbb{R}$, is defined as

$$
V \triangleq \frac{1}{2} z^{\mathrm{T}} M z
$$

Since $M$ is positive-definite and symmetric, the expression in (27) can be lower and upper bounded by the following inequalities

$$
\lambda_{\min }\|z\|^{2} \leq V(z) \leq \lambda_{\max }\|z\|^{2}
$$

where $\lambda_{\min }$ and $\lambda_{\max }$ are the minimum and maximum eigenvalues of $M$, respectively. After taking the first time derivative of (27) and substituting (12), the following expression is obtained

$$
\begin{aligned}
& \dot{V}=z^{\mathrm{T}}\left[f-\hat{f}_{s}-K z-\frac{\rho_{z}^{2}}{\varepsilon} z\right] \\
& \dot{V}=-K z^{\mathrm{T}} z-\frac{\rho_{z}^{2}}{\varepsilon} z^{\mathrm{T}} z+z^{\mathrm{T}} f-z^{\mathrm{T}} \hat{f}_{s}
\end{aligned}
$$

By referring to Remarks 2 and 5 , the function $\dot{V}(t)$ can be upper bounded as

$$
\dot{V} \leq-K\|z\|^{2}-\frac{\rho_{z}^{2}\|z\|^{2}}{\varepsilon}+\|z\| \rho_{z}+\|z\| \rho_{N}
$$

If $K \triangleq k_{1}+k_{2}$ where $k_{1}, k_{2} \in \mathbb{R}^{+}$are constants, the nonlinear damping argument [3] may be applied to (31) to obtain

$$
\dot{V} \leq-k_{1}\|z\|^{2}+\varepsilon_{\mathrm{o}}+\rho_{z}\|z\|\left[1-\frac{\rho_{z}\|z\|}{\varepsilon}\right]
$$

where $\varepsilon_{\mathrm{o}} \triangleq\left(\rho_{N}^{2} / 4 k_{2}\right)$. Using (28) and the non-linear damping argument in (32), the following relationship can be determined

$$
\dot{V} \leq-\left(k_{1} / \lambda_{\min }\right) V+\varepsilon+\varepsilon_{\mathrm{o}}
$$

From (28) and (33), the term $\|z(t)\|$ can be upper bounded as

$$
\|z(t)\| \leq \sqrt{\beta_{0} \exp \left(-\beta_{1} t\right)+\beta_{2}\left[1-\exp \left(-\beta_{1} t\right)\right]}
$$

where

$\beta_{0} \triangleq \frac{\lambda_{\text {max }}}{\lambda_{\text {min }}}\left\|z\left(t_{0}\right)\right\|^{2}, \quad \beta_{1} \triangleq \frac{k_{1}}{\lambda_{\text {max }}} \quad$ and $\beta_{2} \triangleq \frac{\lambda_{\text {max }}}{k_{1} \lambda_{\text {min }}}\left(\varepsilon+\varepsilon_{\mathrm{o}}\right)$

From (34), it can be shown that $e_{1}(t), r_{2}(t) \in \mathcal{L}_{\infty}$; thus from Remark 4, it is clear that $e_{2}(t), \dot{e}_{2}(t) \in \mathcal{L}_{\infty}$. The expression in (6) can be used along with previous boundedness statements to show that $\beta(t), \dot{X}(t) \in \mathcal{L}_{\infty}$; thus from Assumption 5, it is apparent that $f(\cdot) \in \mathcal{L}_{\infty}$. The expression in (11) can be used along with Remarks 3 and 5 to show that $\tau_{\mathrm{c}}(t) \in \mathcal{L}_{\infty}$. Standard signal chasing arguments can then be used to prove that all signals remain bounded under closed-loop operation. In particular, from (12), $\dot{z}(t), \dot{e}_{1}(t), \ddot{e}_{2}(t) \in \mathcal{L}_{\infty}$. Using Assumption 5, it is clear that $\dot{f}(\cdot) \in \mathcal{L}_{\infty}$. The time derivative of $(11)$ can be used along with Remark 5 to show that $\dot{\tau}_{\mathrm{c}}(t) \in \mathcal{L}_{\infty}$. From the time derivative of (5), it is clear that $\dddot{X}(t) \in \mathcal{L}_{\infty}$. Finally it may be concluded that $\ddot{f}(\cdot) \in \mathcal{L}_{\infty}$ using Assumption 5. The closed-loop system is thus GUUB stable.

\section{Appendix 2: Proof of Lemma 1}

The expression in (17) can be substituted into (20) and then integrated in time to obtain

$$
\begin{aligned}
\int_{t_{0}}^{t} L(\tau) \mathrm{d} \tau= & \int_{t_{0}}^{t} \Delta \dot{\tilde{X}}(\tau)^{\mathrm{T}}\left(\dot{f}(\tau)-\rho_{0} \operatorname{sgn}(\dot{\tilde{X}}(\tau))\right) \mathrm{d} \tau \\
& +\left[\int_{t_{0}}^{t} \frac{d \dot{\tilde{X}}^{\mathrm{T}}(\tau)}{\mathrm{d} \tau} \dot{f}(\tau) \mathrm{d} \tau\right] \\
& -\rho_{0} \int_{t_{0}}^{t} \ddot{\tilde{X}}^{\mathrm{T}}(\tau) \operatorname{sgn}(\dot{\tilde{X}}) \mathrm{d} \tau
\end{aligned}
$$




\section{www.ietdl.org}

The bracketed term in (35) may be integrated by parts so that the simplified expression becomes

$$
\begin{aligned}
\int_{t_{0}}^{t} L(\tau) \mathrm{d} \tau= & \int_{t_{0}}^{t} \Delta \dot{\tilde{X}}(\tau)^{\mathrm{T}}\left(\dot{f}(\tau)-\frac{\ddot{f}(\tau)}{\Delta}-\rho_{0} \operatorname{sgn}(\dot{\tilde{X}}(\tau))\right) \mathrm{d} \tau \\
& +\dot{\tilde{X}}(t) \dot{f}(t)-\dot{\tilde{X}}\left(t_{0}\right) \dot{f}\left(t_{\mathrm{o}}\right) \\
& -\rho_{0}\|\dot{\tilde{X}}(t)\|_{1}+\rho_{0}\left\|\dot{\tilde{X}}\left(t_{0}\right)\right\|_{1}
\end{aligned}
$$

The expression in (36) can be upper bounded as

$$
\begin{aligned}
\int_{t_{0}}^{t} L(\tau) \mathrm{d} \tau \leq & \int_{t_{0}}^{t} \Delta\|\dot{\tilde{X}}(\tau)\|_{1}\left(\|\dot{f}(\tau)\|+\frac{\|\ddot{f}(\tau)\|}{\Delta}-\rho_{0}\right) \mathrm{d} \tau \\
& +\|\dot{\tilde{X}}(t)\|_{1}\left(\|\dot{f}(t)\|-\rho_{0}\right) \\
& +\rho_{0}\left\|\dot{\tilde{X}}\left(t_{0}\right)\right\|_{1}-\dot{\tilde{X}}\left(t_{0}\right)^{\mathrm{T}} \dot{f}\left(t_{0}\right)
\end{aligned}
$$

From (37), if

$$
\rho_{0}>\|\dot{f}(\cdot)\|+\frac{\|\ddot{f}(\cdot)\|}{\Delta}
$$

then Lemma 1 holds.

\section{Appendix 3: Proof of Theorem 2}

Define an auxiliary function $P(t) \in \mathbb{R}$ as follows

$$
P \triangleq \zeta_{\mathrm{o}}-\int L(\tau) \mathrm{d} \tau
$$

where $\zeta_{\mathrm{o}}(t)$ and $L(t)$ have been defined in Lemma 1. From the proof of Lemma 1, it is clear that $P(t)$ is non-negative. The following non-negative Lyapunov function, denoted by $V_{1}(t)$, is defined as

$$
V_{1}(t) \triangleq \frac{1}{2} \dot{\tilde{X}}^{\mathrm{T}} \dot{\tilde{X}}+\frac{1}{2} r^{\mathrm{T}} M r+P
$$

After taking the first time derivative of (39), utilising the definitions in (17), (18), (20) and (38), the following expression can be obtained

$$
\dot{V}_{1}=\dot{\tilde{X}}^{\mathrm{T}}(r-\Delta \dot{\tilde{X}})+r^{\mathrm{T}}(-\dot{\tilde{f}}+\Phi-\dot{\tilde{X}})-r^{\mathrm{T}}\left(\dot{f}-\rho_{0} \operatorname{sgn}(\dot{\tilde{X}})\right)
$$

The expression in (40) can be rewritten as

$$
\dot{V}_{1}=-\Delta \dot{\tilde{X}}^{\mathrm{T}} \dot{\tilde{X}}-r^{\mathrm{T}} \dot{\hat{f}}+r^{\mathrm{T}} \Phi+r^{\mathrm{T}} \rho_{0} \operatorname{sgn}(\dot{\tilde{X}})
$$

where (16) was utilised. After substituting (19) and performing simple algebraic manipulations, $\dot{V}_{1}(t)$ can be upper bounded by

$$
\dot{V}_{1} \leq \Delta\|\bar{z}\|^{2}+\left[\|r\| \bar{\rho}_{N}\|\bar{z}\|-k\|r\|^{2}\right]
$$

where $\bar{z}(t)$ is a composite error vector previously defined in Remark 6. By applying the non-linear damping argument [23] to the bracketed term, the expression in (42) becomes

$$
\dot{V}_{1} \leq-\left[\Delta-\frac{\bar{\rho}_{N}^{2}}{4 k}\right]\|\bar{z}\|^{2}
$$

From (43), it is possible to state that

$$
\dot{V}_{1} \leq-\gamma\|\bar{z}\|^{2}
$$

for $k>\left(\bar{\rho}_{N}^{2} / 4 \Delta\right)$ where $\gamma \in \mathbb{R}^{+}$is a constant. From (39) and (44), it is clear that $\bar{z}(t) \in \mathcal{L}_{\infty}$. From the definition of $\bar{z}(t)$, it is clear that $\dot{\tilde{X}}(t), r(t) \in \mathcal{L}_{\infty}$. From (19), it is clear that $\dot{\hat{f}}(\cdot) \in$ $\mathcal{L}_{\infty}$. Using standard signal chasing arguments, it can be shown that all the signals in the closed-loop system remain bounded. In particular, from (18), it may be concluded that $\dot{r}(t) \in \mathcal{L}_{\infty}$. Next, one can deduce that $\dot{\bar{z}}(t) \in \mathcal{L}_{\infty}$. After employing a corollary to Barbalat's Lemma [24], it can be shown that $\|\bar{z}(t)\| \rightarrow 0$ as $t \rightarrow \infty$. From the definition of $\bar{z}(t)$, it is clear that $\dot{\tilde{X}}(t), r(t) \rightarrow 0$ as $t \rightarrow \infty$. From (17), it may be noted that $\ddot{\tilde{X}}(t) \rightarrow 0$ as $t \rightarrow \infty$. From (13) and (14), the following relationship can be obtained

$$
M \ddot{\tilde{X}}=f-\hat{f}=-\tilde{f}
$$

From (44), $\tilde{f}(t) \rightarrow 0$ as $t \rightarrow \infty$, which implies that $\hat{\tau}_{\text {aero }}(t) \rightarrow \tau_{\text {aero }}(t)$. 
Copyright of IET Control Theory \& Applications is the property of Institution of Engineering \& Technology and its content may not be copied or emailed to multiple sites or posted to a listserv without the copyright holder's express written permission. However, users may print, download, or email articles for individual use. 